\title{
Ecoulement à phase liquide dispersée : application à la modélisation de la combustion multiphasique dans les moteurs aéronautiques
}

\author{
Flow with a dispersed liquid phase : application to multiphase combustion \\ in aeronautics engines
}

par D. Bissières, G. Lavergne

DMAE, ONERA/CERT,

Two-phase flow modelling in combustion chambers is based on the Euler-Lagrange approach, respectively for the continuous and dispersed phases. Different models linked to the behavior of a liquid fuel droplet have been developped, and integrated in a numerical simulation module. These models concern droplet turbulent dispersion, evaporation, droplet-wall interactions, secondary breakup, auto-ignition, and interactions between the two phases (liquid and gas). This two-phase flow module now permits to calculate numerous configurations, from ramjets to turboreactors, as well as for space propulsion, internal combustion engines, or any industrial burner. Several aspects have to be furthermore developped, and concern mainly atomisation and dense zones in two-phase flows.

\section{I — CONTEXTE}

L'unité Multiphasique Hétérogène $(\mathrm{MH})$ du Département Modèles pour l'Aérodynamique et l'Energétique (DMAE) de I'ONERA/CERT conduit des recherches sur la modélisation des phénomènes physiques intervenant dans la combustion diphasique. L'injection du carburant sous la forme liquide doit être prise en compte afin de simuler de manière adéquate et précise l'écoulement réactif à l'intérieur des chambres de combustion. Le DMAE/MH élabore des modèles à partir d'expériences de base, l'ensemble de ces résultats a permis de bâtir un module diphasique lagrangien (LSD : Lagrangian Simulation of Droplets $[1,2,3]$ ) comprenant différents modèles élémentaires (dispersion turbulente, évaporation, interaction goutte-paroi, pulvérisation secondaire de goutte, etc.) (figure 1). Ce module LSD peut s'inté grer à différents codes de calcul de la phase continue. En particulier, il a été intégré au DMAE dans une version du code MATHILDA développée à l'ONERA. Cette recherche a de nombreuses applications dans le domaine des chambres de combustion aéronautiques, en particulier pour les statoréacteurs ou les turboréacteurs, ainsi que pour la propulsion spatiale, les moteurs à combustion interne, ou tout autre foyer industriel.

L'objet de cet article est avant tout de faire un état de l'art de la modélisation des écoulements diphasiques à phase dispersée rencontrés en propulsion, et de mettre en évidence les problèmes encore non résolus pour lesquels un effort particulier doit être effectué.

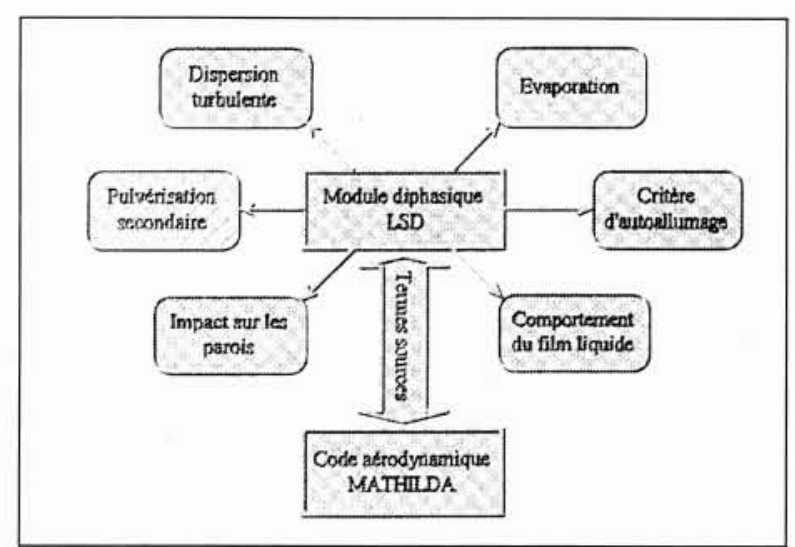

1. Méthodologie de calcul, organigramme du module diphasique LSD.

\section{II — INTRODUCTION}

Les recherches conduites à l'ONERA/CERT/DMAE concernent les écoulements diphasiques à phase dispersée. Pour ces écoulements, la phase continue est composée d'air, et la phase dispersée est formée de gouttes de liquide. Les différents processus physiques liés à la présence de gouttelettes peuvent être décrits chronologiquement depuis l'injection jusqu'à la combustion (figure 2). Tout d'abord, le jet liquide continu issu des injecteurs sera pulvérisé en un brouillard de gouttes, c'est l'atomisation. Ensuite, au sein de 


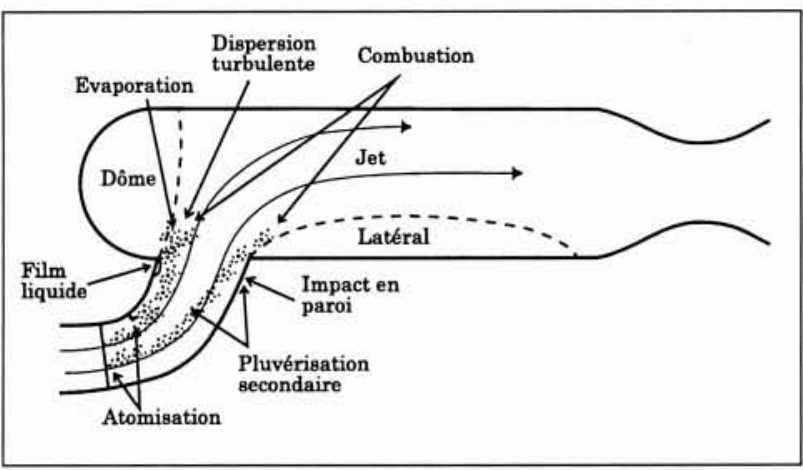

2. Phénomènes physiques relatifs à la phase liquide dans une chambre de combustion de statoréacteur.

ce brouillard, les gouttes interagiront (coalescence), tout en étant transportées par l'écoulement gazeux. Par la suite, lorsque la densité de gouttes devient plus faible, le comportement de ces dernières se rapprochera de celui d'une goutte isolée. On pourra alors considérer le mouvement d'une goutte, en particulier sa dispersion par les structures turbulentes, son évaporation, l'interaction avec une paroi et la pulvérisation secondaire par les forces aérodynamiques.

La réalisation d'expériences de base mais aussi de plus grande complexité permet de développer et de valider des modèles physiques relatifs à la phase liquide, en étudiant les différents processus qui ont été mentionnés plus haut.

Pour la modélisation des écoulements à phase dispersée, l'approche qui est retenue au DMAE est une approche eulérienne lagrangienne. L'écoulement gazeux est considéré comme une phase continue, simulée avec une approche eulérienne. La phase liquide est composée d'un ensemble de gouttelettes, dont on calcule les trajectoires avec une approche lagrangienne. Cette modélisation est adaptée pour le type de configurations étudiées, où la phase dispersée peut être considérée comme diluée. De plus, elle permet une richesse de modélisation basée sur l'étude du comportement d'une goutte isolée qui est directement utilisable.

\section{MODÉLISATION DE LA PHASE LIQUIDE}

\section{- 3.1 Atomisation}

Dans la modélisation de la phase liquide, le phénomène d'atomisation est prépondérant; il constitue en effet la première phase correspondant à la formation des gouttes. Les caractéristiques du brouillard de gouttes formé sont déterminantes puisqu'elles représentent les conditions initiales de la

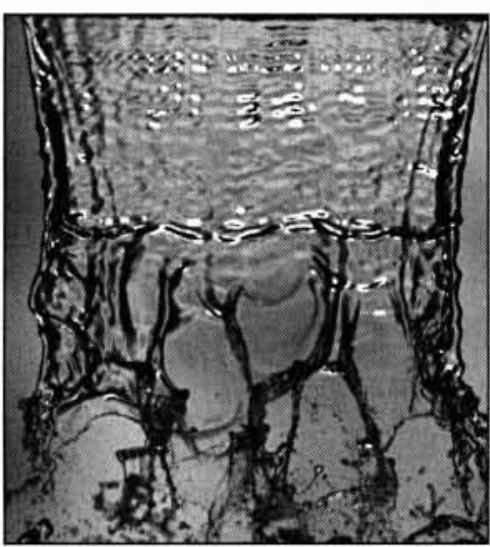

phase liquide. La complexité de ce processus physique rend la modélisation très ardue, et à ce jour, aucun modèle satisfaisant ne peut être utilisé. De nombreuses études visant à améliorer la compréhension des phénomènes physiques sont réalisées actuellement. On peut par exemple citer les travaux sur l'instabilité de nappes liquides avant sa désintégration, à partir d'une approche de stabilité linéaire [4]. Un exemple de visualisation de la désintégration d'une nappe liquide plane est donné sur la figure 3 . On peut observer les différents types d'instabilités présents sur ce type de configurations, qui conditionnent la pulvérisation de la nappe en gouttes. D'autres travaux, basés sur le formalisme de maximum d'entropie sont aussi réalisés [5]. Cette méthode reste encore très empirique, à cause de ses nombreux degrés de liberté. On peut enfin citer de nombreuses corrélations empiriques qui permettent d'obtenir des résultats sur des grandeurs moyennes du brouillard de gouttelettes [6].

Dans l'état actuel des connaissances de l'atomisation, la prédiction des caractéristiques d'un brouillard issu d'un injecteur est impossible. Un effort de recherche important devra être consacré à ce thème, surtout sachant l'importance cruciale de ces caractéristiques qui représentent les conditions initiales de toute simulation d'écoulement diphasique. L'utilisation de données expérimentales détaillées (obtenues par technique Phase-Doppler) permet de contourner cet obstacle, mais sont à l'heure actuelle trop coûteuses pour envisager une utilisation industrielle.

\section{- 3.2 Dispersion turbulente}

Après leur formation, les gouttes sont transportées et dispersées par l'écoulement turbulent instationnaire. Les effets de la turbulence sur ce transport doivent être reproduits. De nombreuses études ont été réalisées, et ont montré l'importance des échelles caractéristiques de la turbulence et du temps de relaxation des gouttes sur le phénomène de dispersion $[7,8,9]$. En effet, comme on peut le voir sur la figure 4 , les gouttes peuvent être capturées par les structures turbulentes ou bien les traverser rapidement. La modélisation, qui doit reproduire cette interaction goutte - structure turbulente, dépend fortement de la méthode de simulation de l'écoulement gazeux. Dans le cas d'une simulation instationnaire de l'écoulement turbulent (DNS ou LES) calculant toutes les échelles significatives de l'écoulement, il suffit de déterminer la trajectoire des gouttes à l'intérieur de ces structures. Par contre, avec une modélisation de l'écoulement moyen (approche de type k- $\varepsilon$ ), il faudra recréer les fluctuations turbulentes et instationnaires de vitesse. Différents modèles stochastiques ont été developpés [1]. Les résultats obtenus sur un grand nombre de configurations bidimensionnelles permettent de bien retrouver les caractéristiques moyennes de la dispersion. On peut toutefois noter d'une part le manque de validation des modèles de dispersion sur des configurations tridimensionnelles complexes, et d'autre part la difficulté de modéliser la turbulence par une approche de type $k-\varepsilon$, qui sera aussi une source d'erreurs pour la dispersion des gouttes.

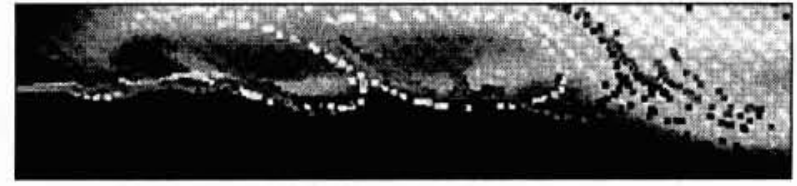

4. Dispersion de gouttes par les structures turbulentes. 


\section{- 3.3 Evaporation}

La modélisation de l'évaporation d'une goutte est étudiée depuis de nombreuses années $[10,11,12]$. Au cours de son évaporation, une goutte subit des échanges de chaleur et de masse avec l'écoulement gazeux. Ces échanges sont très sensibles aux différents paramètres du gaz et du liquide. En particulier, la convection et les propriétés physiques auront une influence significative sur le taux d'évaporation. Différents modèles, basés sur des hypothèses simplificatrices ont été élaborés. Leur validité est directement liée à la vérification de ces hypothèses. On peut par exemple noter, sur la figure 5 , que l'interaction entre les gouttes d'un jet monodisperse modifie radicalement leur évaporation. En effet, les transferts de masse et de chaleur sont diminués à cause de la

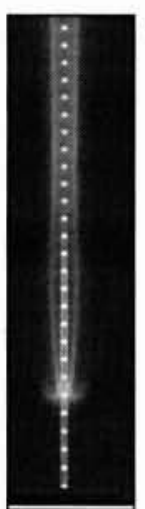

5. Evapora-
tion et
combus-
tion :
flamme de
diffusion
autour
d'un train
de gouttes
monodis-
perses.
proximité des autres gouttes. De plus, dans le cas d'un environnement à haute pression et à haute température, les phénomènes physiques liés au point critique rendent encore plus complexe l'étude de l'évaporation. Enfin, dans le cadre d'application de chambres de combustion, le carburant utilisé est très rarement monoespèce. Il importera alors de modéliser les différents transferts entre les espèces.

\subsection{Interaction goutte paroi}

Les différents régimes d'interaction entre une goutte et une paroi sont très variés, et dépendent majoritairement de la taille, de la vitesse et des propriétés physiques de la goutte, de l'état de la surface de la paroi (température, rugosité), et de la présence ou non d'un film liquide. Les processus physiques observés peuvent être classés en fonction de plusieurs paramètres adimensionnels, tels que le nombre de Weber d'impact, le nombre d'Ohnesorge, le paramètre de surchauffe, et les paramètres de rugosité et d'épaisseur de film $[13,14,15]$. Le rebond, qui sera surtout observé dans le cas d'une paroi chaude, le dépôt partiel ou total seront les principaux régimes d'interaction. Différents modèles ont été élaborés à partir des caractérisations expérimentales de

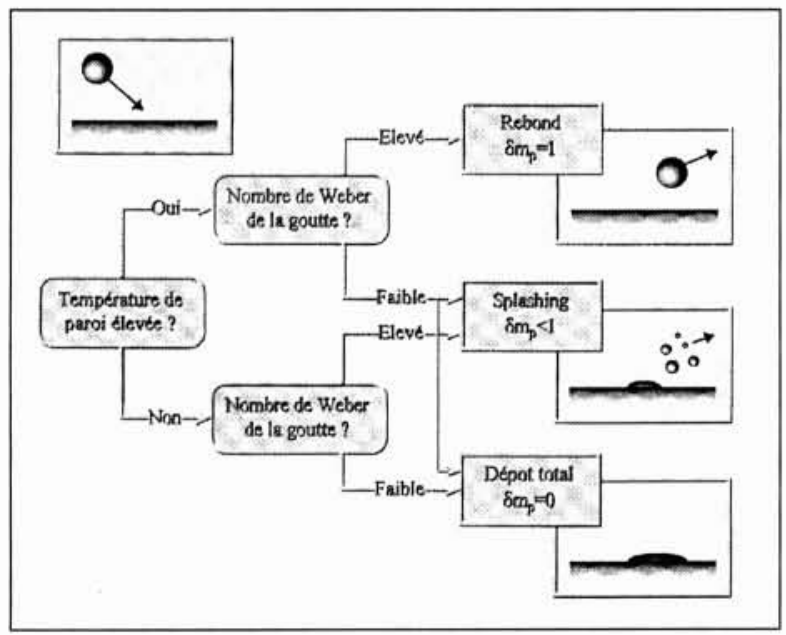

6. Régimes d'impact de gouttes.
l'ONERA/CERT [16]. Le comportement d'une goutte isolée rencontrant une paroi chaude et sèche est relativement bien modélisé, à partir du synoptique présenté sur la figure 6. Par contre, des travaux de recherche doivent être poursuivis pour prendre en compte la présence d'un film liquide, et simuler son comportement en paroi. En particulier, le phénomène d'arrachage de gouttes par le cisaillement de l'écoulement gazeux est un point qui peut être crucial pour la connaissance de la répartition du liquide dans un système d'injection et dans une chambre de combustion (remontée de flamme, inflammation spontanée).

\subsection{Jets denses}

L'hypothèse d'écoulement diphasique dilué sert de base pour l'approche Euler/Lagrange. On peut noter que cette hypothèse pourra être mise en défaut dans certaines zones d'une chambre de combustion. En particulier, près des injecteurs, la densité du brouillard de gouttes est très élevée, ce qui se traduit par de nouveaux phénomènes physiques liés aux interactions entre gouttes. Les principaux aspects à prendre en compte sont alors les collisions entre gouttes, qui conduisent à une augmentation du phénomène de pulvérisation secondaire, et les modifications des régimes d'évaporation et de transport des gouttes. La complexité de ces phénomènes rend les modélisations difficiles, certains aspects pouvant être abordés à partir d'expériences de base telles que l'étude de la collision de gouttes (figure 7), puis intégrés dans des codes de simulation [17]. D'autres aspects, comme la modélisation de l'influence du sillage des gouttes autour d'une goutte de référence sur la traînée et sur le taux d'évaporation, sont encore difficiles à appréhender, même si des corrélations peuvent être dégagées [18].

L'augmentation de la taille des gouttes induite par de nombreuses collisions entraîne une pulvérisation due aux déformations importantes de ces grosses gouttes [19]. L'influence de l'écoulement gazeux se manifeste par l'apparition de différents régimes de pulvérisation, suivant le mode d'instabilité de la sphère de liquide qui est excité. Un exemple de régime de pulvérisation est montré sur la figure 8 . On peut noter ici que l'hypothèse de goutte sphérique indéformable n'est en général pas réaliste, de même que les hypothèses retenues pour modéliser la pulvérisation (symétrie axiale).

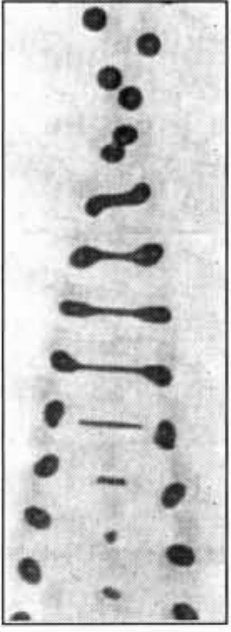

7. Collision de deux trains de gouttes monodisperses.
Pour la modélisation d'un jet dense, un point plus fondamental est la prise en compte du volume occupé par la phase dispersée par rapport au volume total. Une méthode ne reposant plus uniquement sur l'approche lagrangienne permettrait de modéliser la zone proche de l'injecteur, en considérant la phase dispersée avec une approche de milieu continu (approche eulérienne [20]). Une simulation mixte pourrait donc modéliser à la fois les zones denses et diluées d'un écoulement diphasique. 


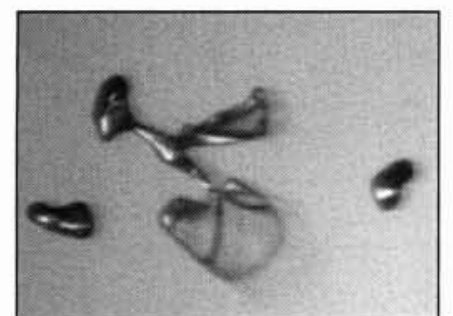

8. Déformation de gouttes induite par l'écoulement et pulvérisation secondaire.

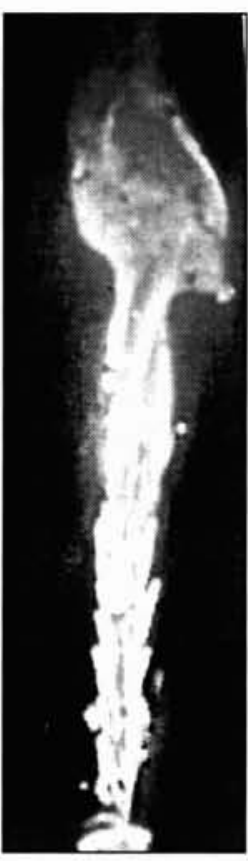

. Combustion en régime critique.

\subsection{Combustion de gouttes}

Suivant la densité des gouttes présentes dans l'écoulement, différents régimes de combustion peuvent intervenir, depuis la combustion de goutte isolée jusqu'à la combustion en paquets. La distance moyenne entre les gouttes sera un des paramètres prépondérants sur le taux de combustion et la forme de la flamme. La turbulence de l'écoulement gazeux aura aussi une influence notable sur la stabilité du front de flamme. Les deux approches de modélisation qui sont actuellement retenues sont basées d'une part sur la combustion d'une goutte isolée, et d'autre part sur l'hypothèse d'une combustion en phase gazeuse. On peut mentionner que la combustion sera bien sûr très sensible aux conditions extérieures, et à l'état des gouttes. En particulier, comme on peut le constater sur la figure 9 , la combustion de gouttes en régime critique présentera des caractéristiques totalement différentes d'une configuration en conditions ambiantes (cf. figure 5). De nombreux efforts sont encore à réaliser sur ce point particulier.

\section{IV — INTERACTIONS ENTRE PHASES}

La modélisation des interactions entre phases est basée sur la détermination des échanges de masse, quantité de mouvement et d'énergie entre les gouttes et l'écoulement porteur (cf. figure 10). Ces échanges sont pris en compte par l'ajout de termes sources au second membre des équations de conservation de la phase gazeuse [21]. Les méthodes numériques de résolution de ces équations doivent donc être adap-

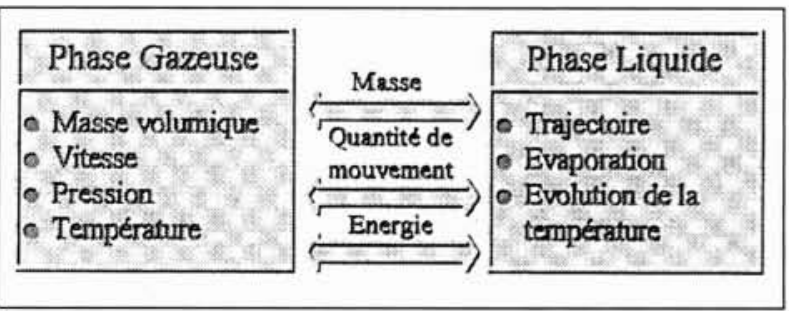

10. Bilan des échanges entre les phases. tées [22], afin d'assurer d'une part une convergence rapide des simulations, et d'autre part d'éviter une divergence par l'apparition de termes sources (par exemple, la présence d'un taux d'évaporation élévé induit un terme source de masse très important). Une méthode basée sur une introduction progressive des termes sources (simulation simultanée des deux phases) peut être envisagée, et permet d'obtenir des résultats rapidement. Un point qui est encore controversé concerne les simulations turbulentes avec une approche de type k- $\varepsilon$. L'ajout de termes sources pour les équations de conservation des grandeurs turbulentes pose des problèmes tant théoriques (comment modéliser l'influence des gouttes sur ces grandeurs) que numériques (divergence causée par la conservation de la grandeur $\varepsilon$ ). On peut noter différents travaux qui essaient d'améliorer ce point $[23,24]$.

\section{APPLICATIONS}

Un premier exemple d'applications concerne la modélisation de l'écoulement diphasique à l'intérieur d'un foyer de statoréacteur [2]. La configuration étudiée est un élargissement brusque axisymétrique (dump). L'injection de carburant (en l'occurence du kérosène) est réalisée, en amont du décrochement, près de la paroi et sur l'axe. Il importe de bien modéliser la dispersion et l'évaporation des gouttes, afin de savoir quelle est la répartition du carburant gazeux dans la chambre. La figure 12 montre l'inhomogénéité de la richesse locale, qui est liée à la dispersion des gouttes. La simulation de l'écoulement réactif pose encore des problèmes, du fait de cette forte inhomogénéité de richesse.

Le deuxième exemple porte sur un module de prémélange pauvre destiné à des chambres de combustion de turbomachines à faible taux de polluants [25]. L'injection de carburant dans ce module doit permettre d'obtenir des conditions optimales dans la chambre, par évaporation du liquide et mélange du carburant. Le fort taux d'évaporation près des parois peut causer localement une réduction importante de la température (la chaleur nécessaire à la vaporisation du

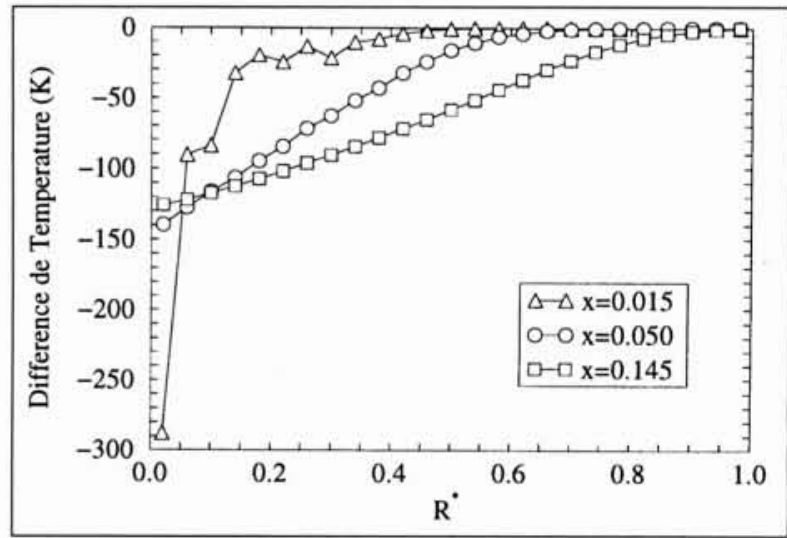

11. Réduction de la température dans un module de prémélange pauvre.

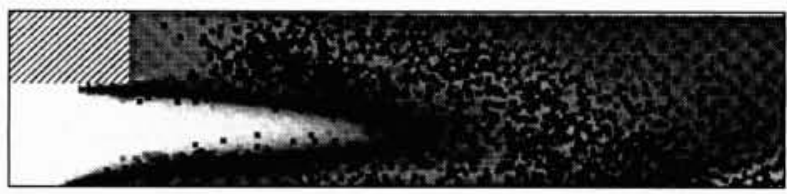

12. Champ de richesse et position des gouttes dans une chambre de combustion de type dump. 


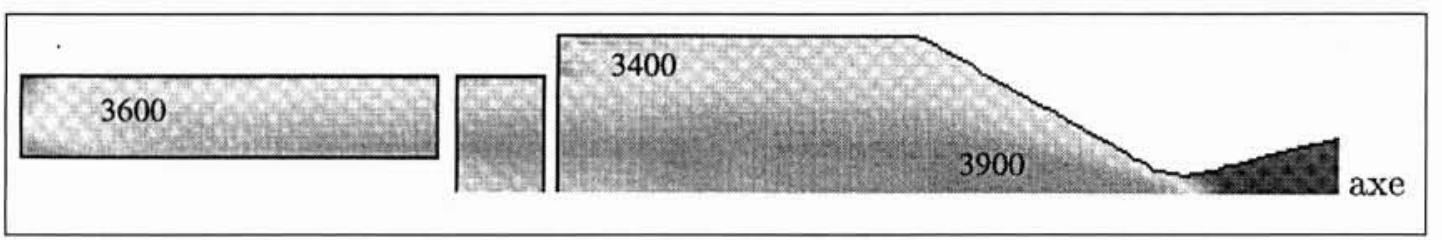
13. Champ de tempéra- ture dans un moteur à proper- gol solide.

liquide est fournie par l'écoulement). Une configuration simple axisymétrique composée d'une partie cylindrique puis conique a été étudiée en simulation. Sur la figure 11 est représentée l'évolution de profils radiaux de température le long du tube. On peut constater que la diminution de la température peut atteindre $300 \mathrm{~K}$ près de l'injection, et reste de l'ordre de $100 \mathrm{~K}$ en sortie du module. Cette configuration nécessite une modélisation très fine de l'impact et du comportement du film liquide en paroi.

Cette approche a enfin été appliquée à un propulseur à propergol solide à échelle réduite [3]. Dans ce cas, la phase liquide est composée d'aluminium, qui s'oxyde en alumine. La modélisation de la combustion de l'aluminium permet alors de déterminer la répartition de température donnée par la combustion distribuée. Un exemple de résultat est donné sur la figure 13 , où l'on peut observer des zones plus chaudes près de l'axe, dues à une forte fraction massique de gouttelettes d'aluminium dans cette zone.

\section{VI $\square$ CONCLUSION}

En conclusion, on peut identifier des points positifs et des points à améliorer :

L'intérêt de l'approche lagrangienne par rapport à l'approche eulérienne réside surtout dans la modélisation plus fine de la phase liquide dans un écoulement diphasique, en particulier au voisinage des parois. La richesse de la modélisation repose sur différents modèles développés pour une goutte isolée. L'atomisation et la partie à forte densité de gouttes proche de l'injecteur sont des domaines encore mal connus. Un effort de recherche soutenu doit être consacré à ce sujet, afin de permettre une modélisation plus réaliste. D'un point de vue plus général, toute zone dense dans un écoulement diphasique est très difficile à simuler. En effet, dans ce cas, on ne peut plus négliger les interactions entre particules et surtout le couplage fort entre les phases (modulation de la turbulence). Un travail spécifique doit être réalisé sur la combustion multiphasique. L'hypothèse de combustion en phase gazeuse est rarement vérifiée, et l'on doit donc prendre en compte les différents régimes de combustion et les carburants multi-espèces.

\section{Références}

[1] P. BÉARD. Modélisation lagrangienne de la dispersion et de l'évaporation de gouttes dans un écoulement turbulent instationnaire. Thèse de Doctorat, ENSAE, 1994.

[2] D. BisSIERES. Modélisation du comportement de la phase liquide dans les chambres de combustion de statoréacteurs. Thèse de Doctorat, Ecole Centrale Paris, 1997. A paraitre.

[3] N. Cesco. Etude de l'écoulement diphasique à l'intérieur des propulseurs à poudre. Thèse de Doctorat, ENSAE, 1997. A paraître.

[4] P. Berthoumieu, P. Carentz, et G. Lavergne Study of planar liquid sheet disintegration. Florence, Italie, 1997. 12 th ILASS.
[5] R.W. Sel.leNs et T.A. Brzustowski. A simplified prediction of droplet velocity distributions in a spray. Combustion and Flame, 65:273-279, 1986.

[6] A.W. LeFEBVRE. Gas turbine combustion. Mac Graw Hill, 1983.

[7] W.H. SNYDER et J.L. LUMLEY. Some measurements of particle velocity autocorrelation functions in a turbulent flow. Journal of Fluid Mechanics, 48(Part 1):41-71, 1971

[8] D.E. STock. Particle dispersion in flowing gases. Journal Fluids Engineering, 118:4-17, 1996.

[9] P. Trichet, G. LAVergne, et Y. Biscos. Amplitude and temporal response of droplets in a turbulent flow field. Dans Proceedings of the 30th AIAA/ASME/SAE/ASEE Joint Propulsion Conference, numéro AIAA-943279. Indianapolis, Indiana, U.S.A., 1994.

[10] G.M. FAETH. Spray atomization and combustion. Dans Proceedings of the 24th Aerospace Sciences Meeting, numéro AIAA 86-0136, Reno, NV, 1986.

[11] C.K. Law. Some recent advances in droplet combustion. Technical report, American Institute of Physics, 1989.

[12] W.A. SiRıgnano. Fluid dynamics of sprays. Journal Fluids Engineering, 115:345-378, 1993 .

[13] L.H.J. WACHTERS et N.A.J. WESTERLING. The heat transfer from a hot wall to impinging water drops in the spheroidal state. Chemical Engineering Science, 21:1047-1056, 1966.

[14] S. DEB et S.-C. YAO. Analysis on film boiling heat transfer of impacting sprays. International Journal of Heat and Mass Transfer, 32(11):2099. $2112,1989$.

[15] C. Mundo et M. SOMMERFEL. Droplet-wall collisions : experimental studies of the deformation and break-up processs. International Journal of Multiphase Flow, 21(2), 1995.

[16] G. Lavergne, F. Bismes, et B. Platet, Etude de l'impact de gouttes sur paroi chauffante. Technical Report RT 2401, CERT/DERMES, 1991.

[17] P. VILLEDIEU et Y. HYLKEMA. Une méthode particulaire aléatoire reposant sur une équation cinétique pour la simulation numérique des sprays denses de gouttes. Comptes Rendus de l'Académie des Sciences de Paris. 325(1):323-328, 1997.

[18] O. ADAM. Etude expérimentale du comportement des gouttes en régime d'interaction. $\mathrm{PhD}$ thesis, ENSAE, 1997.

[19] M. PICLH et C.A. ERDMAN. Use of breakup time data and velocity history data to predict the maximum size of stable fragments for accelerationinduced breakup of a liquid drop. International Journal Multiphase Flow, 13(6): $741-757,1987$

[20] J. Dupays. Contribution à l'étude du rôle de la phase condensée dans la stabilité d'un propulseur à propergol solide pour lanceur spatial. Thèse de Doctorat, I.N.P. Toulouse, 1996.

[21] C.T. Crowe, M.P. Sharama, et D.E. Stock. The Particle-Source-in Cell (PSI-Cell) - model for gas-droplet flows. ASME Journal of Heat Transfer, pages $325-332$, 1975 .

[22] G. KOHNEn, M. Ruger, et M. SOMMERFEL. Convergence behaviour for numerical calculations by the Euler/Lagrange method for strongly coupled phases. Dans Proceedings of the 1994 ASME Fluids Engineering Division Summer Meeting, volume 185, pages 191-202, Lake Tahoe. Nevada, U.S.A., 1994.

[23] A. Berlemont, G. Govesbet, et M.S. Grancher. On the lagrangian simulation of turbulence influence on droplet evaporation. International Journal Heat Mass Transfer, 34(11):2805-2812, 1991.

[24] S. Elghobash et G.C. Truesdel.L. On the two-way interaction between homogeneous turbulence and dispersed solid particles. I : Turbulence modification. Physics of Fluids A, 5(7):1790-1801, 1993

[25] D. Bissieres, G. LaVergne, et P. TRichet, A study of the two-way coupling modelling for the two-phase flow simulation in a lean premixer prevaporizer module. ICLASS. Seoul, Corée, 1997. 